\title{
Combined sphere-spheroid particle model for the retrieval of the microphysical aerosol parameters via regularized inversion of lidar data
}

\author{
Stefanos Samaras ${ }^{1}$, Christine Böckmann $^{1}$, Doina Nicolae ${ }^{2}$ \\ ${ }^{1}$ University of Potsdam, Am Neuen Palais 10, 14469 Potsdam, Germany,samaras@uni-potsdam.de, \\ Germany,bockmann@rz.uni-potsdam.de \\ ${ }^{2}$ Laser Remote Sensing Department, National Institute of R\&D for Optoelectronics, 409 Atomistilor Str., Magurele, Ilfov, \\ Romania,nnicol@inoe.ro
}

\begin{abstract}
In this work we propose a two-step advancement of the Mie spherical-particle model accounting for particle nonsphericity. First, a naturally two-dimensional (2D) generalized model (GM) is made, which further triggers analogous 2D re-definitions of microphysical parameters. We consider a spheroidal-particle approach where the size distribution is additionally dependent on aspect ratio. Second, we incorporate the notion of a sphere-spheroid particle mixture (PM) weighted by a non-sphericity percentage. The efficiency of these two models is investigated running synthetic data retrievals with two different regularization methods to account for the inherent instability of the inversion procedure. Our preliminary studies show that a retrieval with the PM model improves the fitting errors and the microphysical parameter retrieval and it has at least the same efficiency as the GM. While the general trend of the initial size distributions is captured in our numerical experiments, the reconstructions are subject to artifacts. Finally, our approach is applied to a measurement case yielding acceptable results.
\end{abstract}

\section{Introduction}

A central topic in understanding the climate processes is the investigation of the spatial and temporal variability of the microphysical properties of aerosol particles, such as the aerosol size distribution, the complex refractive index and the mean particle radius. Recent development of continental-scale lidar networks provides the opportunity to obtain quality assured optical profiles on a large temporal and spatial scale, e.g. the European Aerosol Research Lidar Network (EARLINET) involvement. In particular, Raman-elastic multi-wavelength lidar data is used [1] to measure the aerosol optical properties and to retrieve the size distribution through the inversion of the Mie forward model.

Regularization methods are a widely preferable choice for an efficient inversion, e.g. [2,9]. While the sphericalparticle (Mie) model offers a reasonable approximation for spherically modelled particles, e.g. biomass burning aerosols, it no longer provides a viable description for the more realistic non-spherical case such as mineral dust; particle shape is known to have substantial effects for the scattering in side- and backward direction [8]. Nevertheless the first non-spherical model approximation, i.e. spheroids, seems to reproduce the optical properties of realistic aerosol particles significantly better than spheres $[6,8]$. Steps towards particle non-sphericity incorporation for the purpose of a microphysical retrieval are a result of very recent work mostly considering spheroidalparticle models, e.g. $[3,4,13]$.

In the present work, we use the generalization of the Mie forward model (GM) in two dimensions (2D) and perform simulations with two regularization methods to study the retrieval behavior and compare. We further adopt the concept of a mixture of spherical and spheroidal particles, quantitatively dictated by a nonsphericity percentage, introduced in [7]. The particlemixture model (PM) is then compared to the (GM) with respect to the most appropriate of the regularization methods used previously. Our algorithm is subsequently applied to a measurement case from the Bucharest lidar station. This study is a part of our first attempts to familiarize with potential retrieval patterns of our newly developed software.

\section{Mathematical standpoint: two-dimensional forward models and ill-posedness}

The aerosol volume size distribution $v(r, a)$ is related to the extinction- and backscatter coefficients, $\Gamma(\lambda)$ through a generalization of Mie forward model (GM) in two dimensions and the so-called Fredholm integral equation of the first kind:

$$
\Gamma(\lambda)=\int_{a_{\min }}^{a_{\max }} \int_{\mathrm{r}_{\min }}^{r_{\max }} K_{\alpha / \beta}(r, \lambda, a ; m) v(r, a) d r d a,
$$

where $K_{\alpha / \beta}=\frac{3 S}{16 \pi r^{3}} Q_{\alpha / \beta}$, are the extinction- $(\alpha)$ or backscatter kernels $(\beta)$, summarizing the relation of the scattering efficiencies $Q_{\alpha / \beta}$ with the spheroidal surfacearea $S$. The dependency on the volume-equivalent radius and the aspect ratio is denoted by $r$ and $a$, respectively, and $m$ is the refractive index. In this work the wavelength $\lambda$ is restricted to the discrete values $\lambda_{i}=\{355,532,1064\}$ $\mu \mathrm{m}, i=\{1,2,3\}$. The ideal optical data setup here $(3 \beta+2 \alpha+3 d)$ consists of 3 backscatter coefficients horizontally polarized $\beta_{\lambda_{1,2,3}}, 2$ extinction coefficients $\alpha_{\lambda_{1,2}}$ and 3 vertically polarized backscatter coefficients $d_{\lambda_{1,2,3}}$. The computational effort for the calculation of the kernel function using T-matrix method [8] is very high for realtime retrievals. Instead we use a precalculated database 
produced by Mieschka software tool, see [12]. The refractive index is commonly considered wavelength independent, see e.g. [2]. Moreover, a refractive index grid is predefined, upon which the solution space is built. For the retrieval process, the model is ran for every different value of the grid and solution is picked by means of least residual error.

Eq. 1 belongs to the class of inverse ill-posed problems, see [11]. This means that potential noisy components in the input data $\Gamma(\lambda)$ will result in oscillatory solutions, regardless of the noise level, see e.g. $[2,10]$. The degree of ill-posedness is linked to the level of smoothness of the scattering efficiencies, thus their qualitative behavior indicates where the problem arises. Fig. 1 shows a multiple plot of the total backscatter efficiencies with respect to the size parameter $s=\frac{2 \pi r}{\lambda}$ for different real part of refractive index (RRI) and constant imaginary part of refractive index (IRI) and a fixed aspect ratio; the oscillations are suppressed with decreasing RRI. In Fig. 2, similar trend can be observed for increasing IRI (higher absorption levels) and constant RRI. In particular, looking along the aspect ratio axis of the lower 3D plots in Fig. 2 we observe that extinction efficiencies cannot reveal any shape information for a large IRI in the case of larger size parameters.

Eq. 1 is discretized with spline collocation using variable number $(n)$ and degree $(d)$ of B-splines $\phi_{j, k}^{(d)}$, using the size distribution approximation $v_{p q}=$ $\sum_{j=1}^{p} \sum_{k=1}^{q} b_{j k} \phi_{j k}^{(d)}(r, a)$. Then, an one-dimensional index reordering $L=F(j, k)$ is used to build the matrix $\sum_{L=1}^{p q} A_{i L} b_{L}=\Gamma_{i}, i=1, \ldots, 8$. We solve the resulting linear system with the following regularization methods:

1. TSVD with the Discrepancy Principle (TSVD),

2. Tikhonov with the L-curve method (TLC).

There are well-known essential differences between these methods, but the main one here is that only method 1 makes use of a known error level.

Next, we define the particle mixture model (PM) as follows:

$$
\begin{aligned}
\Gamma(\lambda) & =(1-\eta) \int_{r_{\min }}^{r_{\max }} K_{\alpha / \beta}^{\mathrm{sph}}(r, \lambda, m) v(r) \mathrm{d} r+ \\
& +\eta \int_{a_{\min }}^{a_{\max }} \int_{r_{\min }}^{r_{\max }} K_{\alpha / \beta}^{\mathrm{nsph}}(r, \lambda, m, a) v(r, a) \mathrm{d} r \mathrm{~d} a
\end{aligned}
$$

where the superscript sph/nsph refers to the spherical $(a=1)$ or non-spherical $(a \neq 1)$ kernel functions. The assumption here is that the aerosol particles consist of independent spherical and non-spherical (spheroidal) parts, a property which further passes on the optical data through a non-sphericity percentage we call asphericity $(\eta)$. The solution calculation is slightly modified here. We define a range of viable asphericity options and run (PM) for every value within it and for every refractive index within the grid to finally bring out one least residual error solution.

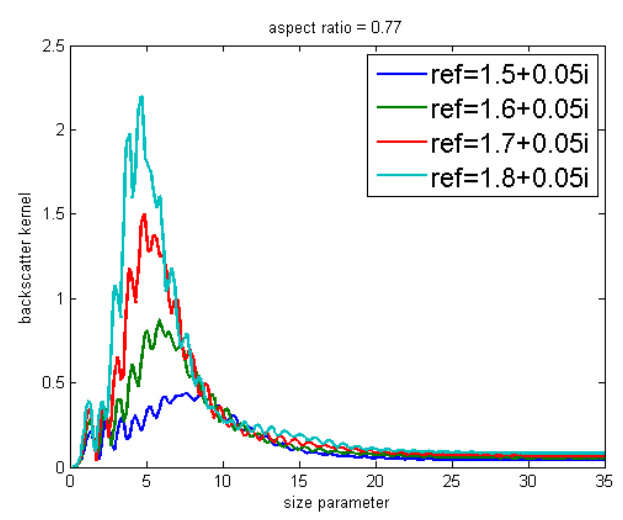

Fig. 1. Total backscatter efficiencies with respect to size parameter for fixed aspect ratio and different RRI.
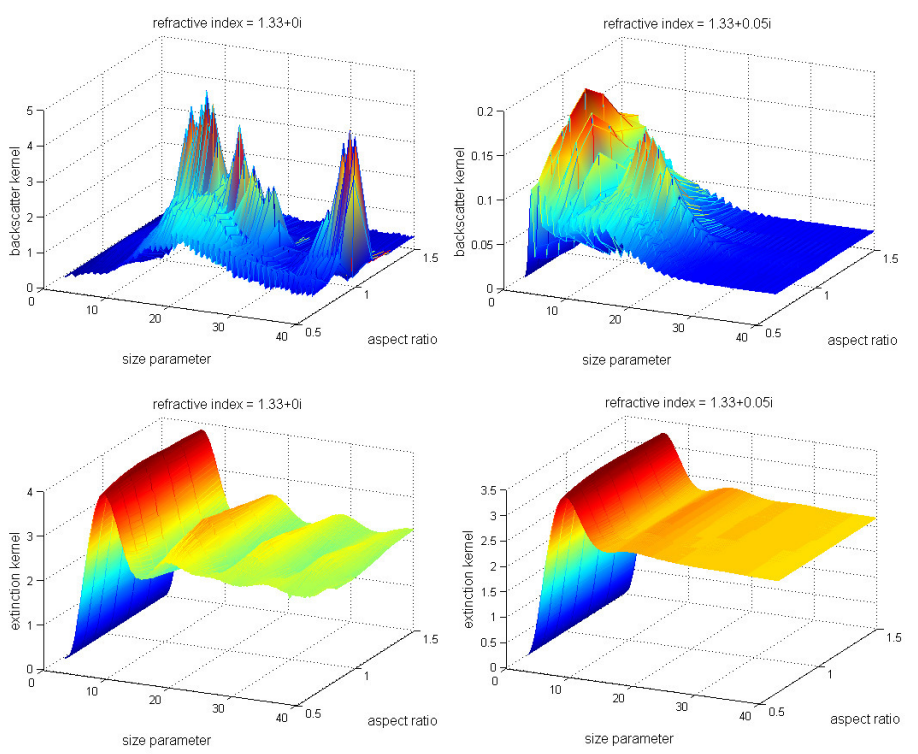

Fig. 2. Total backscatter- (upper panel) and extinction efficiencies (lower panel) for increasing IRI from left to right.

\section{Synthetic data results}

In all the simulations that follow we assume the same lognormally distributed radius range $[0.03,1.24]$ with mean radius $\mu=0.25$, width $\sigma=1.2$ and number concentration $n_{t}=1$ combined with an aspect ratio distribution of a purely oblate particle sample. In this experiment we would like to see the retrieval behavior of the methods $1-$ 2 for the size distributions. The synthetic data were produced with a forward calculation and $3 \%$ random errors were added. We reconstructed $v(r, a)$ with each regularization method using the same spline number and degree $(9,6)$ and a fixed refractive index $m=1.4+0.01 i$. Fig. 3 shows the solution reconstructions with the two methods for the case of an oblate spheroidal sample. Both methods seem to capture the general trend with almost the same efficiency. The artifacts in the case of TSVD are more prominent, and the fitting error (not shown here) 
Table 1. Comparison of the models GM and PM with respect to TLC-retrieved microphysical parameters

\begin{tabular}{|l||l|l|l||l|l|}
\hline Parameters & $\begin{array}{l}\text { Synthetic } \\
\text { values }\end{array}$ & $\begin{array}{l}\text { Solution } \\
(\mathrm{GM})\end{array}$ & $\begin{array}{l}\text { Solution } \\
(\mathrm{GM})\end{array}$ & $\begin{array}{l}\text { Solution 1 } \\
(\mathrm{PM})\end{array}$ & $\begin{array}{l}\text { Solution } \\
(\mathrm{PM})\end{array}$ \\
\hline $\mathrm{m}$ & $1.33+0.1 \mathrm{i}$ & $1.33+0.03 \mathrm{i}$ & $1.33+0.03 \mathrm{i}$ & $1.33+0.05 \mathrm{i}$ & $1.33+0.05 \mathrm{i}$ \\
\hline$n_{t}$ & 0.076 & 0.20 & 0.20 & 0.05 & 0.06 \\
\hline$a_{t}$ & 0.015 & 0.015 & 0.015 & 0.002 & 0.003 \\
\hline$v_{t}$ & 0.006 & 0.005 & 0.005 & 0.0008 & 0.001 \\
\hline$r_{\mathrm{eff}}(v)$ & 1.09 & 0.95 & 0.97 & 0.94 & 0.92 \\
\hline$\eta$ & 0.84 & 0.90 & 0.90 & 0.86 & 0.89 \\
\hline splines no./degree & $100 \%$ & $100 \%$ & $100 \%$ & $47 \%$ & $56 \%$ \\
\hline reconstruction error & - & $22 / 4$ & $21 / 4$ & $22 / 4$ & $21 / 4$ \\
\hline
\end{tabular}

is larger than in the case of TLC. A closer look to the regularization parameter of TSVD, the cut-off level $(\mathrm{cl})$, see [5], for this case reveals that such a behavior is to be expected since the noise is dominating and causes an underestimation of the $\mathrm{cl}$.
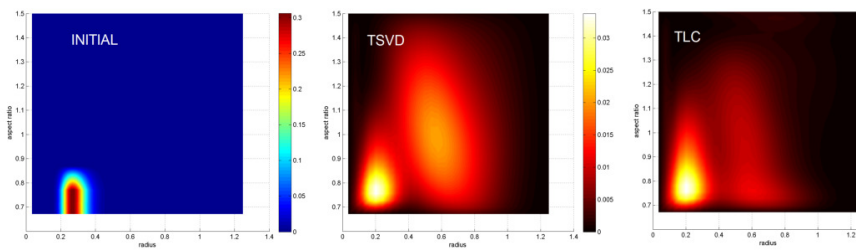

Fig. 3. From left to right: Volume size distribution reconstructions with TSVD and TLC method compared to the initial one (leftmost).

Table 2. Microphysical parameters derived with the method from [2] and the TLC method with the (PM) model for the measurement case captured by the lidar station in Magurele, Bucharest.

\begin{tabular}{|l|l||l|}
\hline Parameters & Method from $[2]$ & TLC - PM \\
\hline $\mathrm{m}$ & $1.35+0.002 \mathrm{i}$ & $1.33+0.005 \mathrm{i}$ \\
\hline$n_{t}$ & 0.0006 & 0.00017 \\
\hline$a_{t}$ & 0.0001 & $8.06 \mathrm{e}-06$ \\
\hline$v_{t}$ & $1.36 \mathrm{e}-05$ & $2.35 \mathrm{e}-06$ \\
\hline$r_{\mathrm{eff}}$ & 0.28 & 0.86 \\
\hline$\mu_{a}^{(v)}$ & - & 0.77 \\
\hline$\eta$ & - & $22.7 \%$ \\
\hline splines no./degree & $7 / 3$ & $9 / 3$ \\
\hline reconstruction error & $1.87 \mathrm{e}-05$ & $9.1 \mathrm{e}-07$ \\
\hline
\end{tabular}

\section{Microphysical parameter retrieval}

First we need some definitions which follow the same concept as the 2D generalization of Eq. 1: the number concentration:

$$
n_{t}=\int_{a_{\min }}^{a_{\max }} \int_{r_{\min }}^{r_{\max }} \frac{3}{4 \pi r^{3}} v(n, a) d r d a,
$$
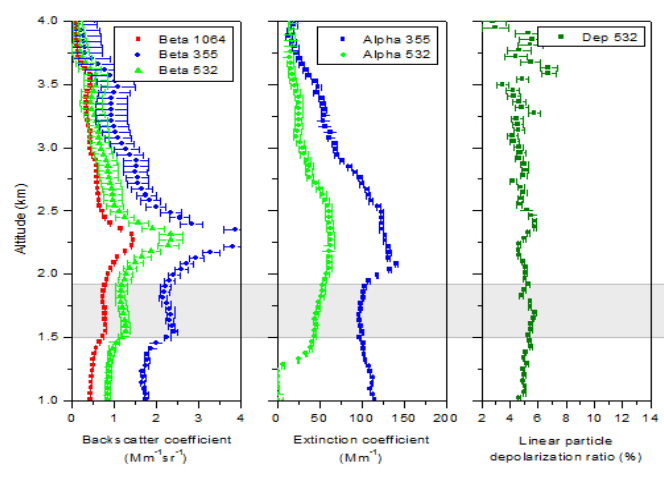

Fig. 4. From left to right: Extinction coefficient, backscatter coefficient and linear depolarization ratio profiles for the measurement case captured by the lidar station in Magurele, Bucharest. Gray-shaded areas highlight the layer values used for retrieving the microphysical properties shown in Table 2.

the total volume concentration:

$$
v_{t}=\int_{a_{\min }}^{a_{\max }} \int_{r_{\min }}^{r_{\max }} v(r, a) d r d a,
$$

the surface-area concentration

$$
a_{t}=\int_{a_{\min }}^{a_{\max }} \int_{r_{\min }}^{r_{\max }} \frac{3}{\pi r^{3}} G(r, a) v(r, a) d r d a,
$$

where $G(r, a)$ is the geometrical cross section of the particle. The effective radius can as usual be defined by $r_{\text {eff }}=\frac{3 v_{t}}{a_{t}}$. Moreover, we define the effective aspect ratio:

$$
\mu_{a}^{(v)}=\frac{\int_{a_{\min }}^{a_{\max }} a \int_{r_{\min }}^{r_{\max }} v(r, a) d r d a}{v_{t}} .
$$

In this experiment we retrieve the microphysical parameters $m, n_{t}, v_{t}, a_{t}, r_{\mathrm{eff}}$, and $\mu_{a}^{(v)}$ using the models GM (Eq. 1) and PM (Eq. 2) with TLC method and the same test-configuration used in section 3 except that now the refractive index resides within the grid $\Re(m) \times \Im(m)$, where $\Re(m)=\{1.33,1.4,1.5,1.6,1.7,1.8\}$, $\Im(m)=\{0,0.001,0.005,0.01,0.03,0.05,0.1\}$, see $[12]$. 
The results are in Table 1; we include two least-residualerror solutions from each run for comparison. We can see that the new model (PM) improves the fitting errors, reproducing the initial optical data better than the (GM). The parameters $m, n_{t}$ and $\mu_{a}^{(v)}$ are better retrieved in $(\mathrm{PM}), r_{\text {eff }}$, are equivalent with both models, while $a_{t}$ and $v_{t}$ are underestimated and better retrieved with (GM). The (PM) model offers additional knowledge on the nonsphericity predicting a percentage of $47 \%$ and $56 \%$ for the two solutions shown. Although there is room for improvements evidently, we will consider (PM) as an enhancement of (GM) model and use this for the measurement case in section 5 .

\section{Retrieval from a measurement case}

In this section we apply our model (PM) to a measurement case captured by the multiwavelength depolarization Raman lidar in Magurele, $6 \mathrm{~km}$ south of Bucharest (lat. 44.348 N, long. 26.029 E, above sea level), on June, 27th 2012 between 19:30-20:30 UTC, see Fig. 4. The HYSPLIT model and MODIS fire maps confirmed that this is probably a biomass burning aerosol case, which is in accordance with the low depolarization value of $5 \%$, suggesting almost spherical particles. In Table 2 we show the microphysical parameters derived in two ways; (1) with an older solution algorithm of our group based on the Mie forward model, presented in [2], and (2) the TLC-(PM) method. A direct comparison requires a deeper analysis, but here we will focus on the orders of magnitude for the retrieved values using TLC$(\mathrm{PM})$, which appear reasonable. The $(\mathrm{PM})$ predicts an atmospheric scenario of particles acting as an oblate spheroidal sample $\left(\mu_{a}^{(v)}=0.77\right)$ with a non-sphericity percentage of $22.7 \%$.

\section{Summary}

We investigated a natural generalization of the Mieforward model (GM) considering a size distribution depending both on the radius and the aspect ratio and subsequently employed both spherical and spheroidal efficiencies and the notion of a non-sphericity percentage to a new model (PM). TLC-retrieved size distributions seem to be less prune to artifacts and yield smaller fitting errors compared to TSVD ones. PM along with the use of TLC indicates that it is a correction to the GM model, offering better fitting errors, and improving the key microphysical parameters, refractive index and number concentration. Finally, the results from the application to a measurement case fall within the orderof-magnitude tolerance and the limitations of this approach.

\section{Acknowledgements}

The research leading to these results has received funding mainly from the European Unions Seventh Framework Programme for research, technological develop- ment and demonstration under grant agreement no 289923 - ITaRS.

\section{References}

1. Ansmann, A., M. Riebesell, U. Wandinger, C. Weitkamp, E. Voss, W. Lahmann, W. Michaelis, 1992: Combined Raman elastic-backscatter lidar for vertical profiling of moisture, aerosol extinction, backscatter, and lidar ratio, Applied Physics B, 55(1), 18-28.

2. Böckmann C., 2001: Hybrid regularization method for the ill-posed inversion of multiwavelength lidar data in the retrieval of aerosol size distributions, Applied Optics, 40(9), 1329-1342.

3. Böckmann C., L. Osterloh, 2014: Runge-kutta type regularization method for inversion of spheroidal particle distribution from limited optical data, Inverse Problems in Science and Engineering, 22, 150-165.

4. Dubovik O., B. N. Holben, T. Lapyonok, A. Sinyuk, M. I. Mishchenko, P. Yang, I. Slutsker, 2002: Non-spherical aerosol retrieval method employing light scattering by spheroids, Geophysical Research Letters, 29(10), 1415-1418.

5. Hansen P. C., 2010: Discrete inverse problems: insight and algorithms, SIAM, 7.

6. Kahnert M., A. Kylling: Radiance and flux simulations for mineral dust aerosols: Assessing the error due to using spherical or spheroidal model particles, Journal of Geophysical Research: Atmospheres, 109(D9), 2156-2202.

7. Lopatin A., O. Dubovik, A. Chaikovsky, P. Goloub, T. Lapyonok, D. Tanré, P. Litvinov: Enhancement of aerosol characterization using synergy of lidar and sunphotometer coincident observations: the GARRLiC algorithm, Atmospheric Measurement Techniques, 6(8), 2065-2088.

8. Mishchenko M. I., L. D. Travis, D. W. Mackowski: Tmatrix computations of light scattering by nonspherical particles: A review, Journal of Quantitative Spectroscopy and Radiative Transfer, 55(5), 535-575.

9. Müller D., U. Wandinger, and A. Ansmann: Microphysical particle parameters from extinction and backscatter lidar data by inversion with regularization: simulation, Applied Optics, 38(12), 2358-2368.

10. Osterloh L., C. Pérez, D. Böhme, J. M. Baldasano, C. Böckmann, L. Schneidenbach, D. Vicente, 2009: Parallel software for retrieval of aerosol distribution from lidar data in the framework of EARLINET-ASOS, Computer Physics Communications, 180(11), 2095-2102.

11. Rieder A., 2003: Keine Probleme mit Inversen Problemen - Eine Einfhrung in ihre stabile Lösung, ViewegTeubner Verlag.

12. Rother T., 2009: Electromagnetic Wave Scattering on Non- spherical Particles: Basic Methodology and Simulations, Springer.

13. Veselovskii I., O. Dubovik, A. Kolgotin, T. Lapyonok, P. Di Girolamo, D. Summa, D. N. Whiteman, M. Mishchenko, D. Tanr, 2010: Application of randomly oriented spheroids for retrieval of dust particle parameters from multiwavelength lidar measurements, Journal of Geophysical Research: Atmospheres, 115(D21). 